\title{
Challenges and coping strategies in non-communicable disease management during COVID-19 in Addis Ababa, Ethiopia: A qualitative study
}

\section{Bezawit Ketema}

Addis Ababa University School of Public Health https://orcid.org/0000-0002-2380-4779

Mirgissa Kaba

Addis Ababa University School of Public Health

Adamu Addissie

Addis Ababa University School of Public Health

Eva Johanna Kantelhardt ( $\sim$ eva.kantelhardt@uk-halle.de)

Martin-Luther-University https://orcid.org/0000-0001-7935-719X

\section{Research article}

Keywords: COVID-19, non-communicable diseases, service disruption, telemedicine.

Posted Date: August 25th, 2020

DOl: https://doi.org/10.21203/rs.3.rs-56009/v1

License: (9) (1) This work is licensed under a Creative Commons Attribution 4.0 International License. Read Full License 


\section{Abstract}

Background People with comorbidities are at higher risk of becoming severely ill with COVID-19. Evidence has shown that those with cardiovascular diseases, chronic respiratory diseases, diabetes and cancer had more severe illness and worse prognosis with COVID19 compared to patients without comorbidities. Insufficient numbers of health workers, high financial barriers and lack of access to quality-assured medicines have been challenges for patients with non-communicable diseases (NCDs) in developing countries, even before COVID-19. Therefore, this study aimed to explore the challenges and coping strategies in NCD management during COVID-19 in Addis Ababa, Ethiopia.

Methods This study followed a phenomenological study approach to explore challenges and coping strategies using the experience of healthcare professionals (HCPs) and people with NCDs. Twenty-two individuals (13 HCPs and nine people with NCDs) were recruited purposively and invited for in-depth interview. All interviews were made by telephone and data collected using interview guide. There was pre-interview telephonic conversation to ask participants for a convenient time and date for the actual interview. At the end of every interview, a summary of the interview notes was read to the participants to ensure the correct interpretation of participants' original views. ATLAS.ti version 7 software was used to assist in the coding and categorisation. Thematic analysis was employed to understand the challenges and coping strategies in NCD management during COVID-19 in Addis Ababa, Ethiopia.

Results Challenges posed by COVID-19 to people with NCD included postponement of NCD follow-up due to fear of COVID-19 infection, transportation problems and unwelcoming healthcare facilities; stress related to fear of death and absence of social support; and reduced physical activity in relation to staying at home. For the HCPs, a shortage of personal protective equipment and an absence of standards and guidelines were the common challenges. Telemedicine was the typical coping strategy used by HCPs, while people with NCDs were mostly expecting spiritual protection and safeguarding.

Conclusions We concluded that the postponement of NCD follow-up, in addition to stress and reduced physical activity, may worsen the increasing mortality from preventable NCDs in the country. We recommend every COVID-19 response measures to target NCD health service independently.

\section{Background}

The COVID-19 pandemic from the beginning of 2020 reached Ethiopia in March 13, 2020. Up to mid-July, 8803 cases and 150 deaths were reported [1].

The current mortality rate from COVID-19 (4.25\%) [2] is relatively low as compared to other emerging viruses such as Severe Acute Respiratory Syndrome (SARS; 9.6\%) and Middle East Respiratory Syndrome (MERS; 34.4\%) [3, 4]. However, people with comorbidities are more exposed to become severely ill with COVID-19 [5]. A report from Italy revealed that the majority (96.2\%) of patients who have died in the hospital from COVID-19 had comorbidities, mostly non-communicable diseases (NCDs); the most prevalent NCDs among these patients were hypertension (69.2\%), type 2 diabetes (31.8\%), ischaemic heart disease $(28.2 \%)$, chronic obstructive pulmonary disease (16.9\%), and cancer (16.3\%) [6]. Evidence from China, Spain, and the USA also exhibited an association between the severity of COVID-19 and the presence of NCDs [7-9].

Non-communicable diseases kill 41 million people each year, equivalent to $71 \%$ of all deaths globally. Each year, 15 million people between the ages of 30 and 69 die from an NCD, with more than $85 \%$ of these 'premature' deaths occurring in low- and middle-income countries [10]. In Ethiopia, $16 \%$ and $5.4 \%$ of the population have raised blood pressure and impaired fasting glycaemia, respectively [11]. Prevalent cancer cases (5-year) totalled 107,644 [12]. Non-communicable diseases contribute to $39 \%$ of total deaths in Ethiopia [13]. Insufficient health workforce and pharmaceuticals, high financial barriers for users and lack of access to quality-assured medicines are the challenges for people with NCDs in developing countries $[14,15]$. The additional burden of the pandemic in countries like Ethiopia might become more demanding, since it has even challenged countries with well-developed healthcare systems [16].

Thus, considering people with NCDs as a high-risk population, special arrangements are required to ensure the availability of routine services without exposing patients to the risk of COVID-19. Therefore, this study explored challenges and coping strategies in NCD management during the COVID-19 pandemic in Addis Ababa, Ethiopia. 


\section{Methods}

\section{Study approach}

We followed a phenomenological study approach to explore the challenges and coping strategies in NCD management during the COVID-19 pandemic in Addis Ababa, Ethiopia, from the lived experience of people with NCDs and healthcare professionals (HCPs) working in NCD management.

\section{Study setting and period}

This study was conducted from 20.04.2020 to 20.05.2020 in Addis Ababa, the capital city of Ethiopia. According to the Ethiopian Central Statistical Agency Population Projection, the city had an estimated population of 3,433,999 in 2017 [17]. In Ethiopia, the highest proportion of morbidities attributed to NCDs are in Addis Ababa [18].

\section{Study participants}

Health care professionals working in the management of NCDs (physicians, nurses and pharmacists) and people with NCDs (hypertension, diabetes and cancer) were recruited purposively with criterion sampling method from both public and private health facilities at primary, secondary and tertiary levels and invited for in-depth-interview. The sample size was determined up to saturation level, where redundancy of information was seen.

\section{Data collection procedure}

Data was collected by one-to-one in-depth interview via telephone using an interview guide. Interview guides were developed by the authors for this study (Additional file 1). Data collectors/interviewers were provided a half day training by the authors. There was pilot testing, by which data collectors/interviewers have been familiar with the interview guides, test their audio recorders and exercise conducting telephonic qualitative data collection by doing pilot test of 1-2 interviews among each other. All interviewers who have conducted the data collection for this study have MPH and very good experience in qualitative data collection. There was preinterview telephonic conversation with study participants to take consent and to ask for a convenient date and time for interview. During the pre-interview telephonic conversation participants were also told to be in a quiet environment for the actual interview. All the interviews were carried out in a quiet environment, with good phone network and in the Amharic language. Six of the 22 study participants were called back as part of the data collection process. Five of them for additional information and 1 of them due to dead mobile phone battery.

All the interviews were tape recorded. In addition interview notes were taken promptly during the interview, which helped in identifying follow-up questions. An interview lasted for 30-45 minutes on average. The interview was maintained flexible enough in terms of participants' responses to questions, without limiting or interrupting. Participants were invited to talk about their challenges in NCD management in relation to COVID-19. Subsequently, they were asked about the coping strategies they were using for the challenges that COVID-19 brought in NCD management. In closing the interview session their responses were summarised and read back to the respondents by the interviewer in order to build rapport. By doing that, misunderstandings were corrected immediately. Finally, after the participants were thanked for their time and cooperation, permission was sought to call back in case of any need for clarification or additional information.

\section{Data quality assurance}

Trustworthiness of the findings was ensured in various ways. For example, at the end of every interview, a summary of interview notes was read to the participants in order to ensure the correct interpretation of participants' original views. A report of the study findings was virtually presented to national and international audiences for examination of the process of data collection, analysis and interpretation [19], where colleagues and some study participants have attended and given feedback. In addition, two coders independently coded similar transcriptions and agreement was observed. Furthermore, transferability of findings was facilitated by providing sufficient information about the research context, methodology and participants.

\section{Data analysis}


Preliminary manual analysis was carried out simultaneously with the data collection. The same day, after finishing the interview session, information stored on mobile recorders was listened to repeatedly and transcribed verbatim by the interviewer and then translated into English. Even though codes emerged in the preliminary manual analysis during the data collection period, the code book was refined after completion of the data collection. Each code was described based on the interviewed participants' ideas from the transcriptions. Then, following thematic analysis, the words of participants and their meanings were used in coding and categorisation using ATLAS.ti 7 software. Themes were not identified from the beginning, rather it emerged from the data. At the first level words or phrases were used to form codes, then similar codes were combined for categorisation. Similar categories were then brought together to form a theme which represented the whole idea of the categories. Finally, word descriptions, narrations, participants' quotations and tables were used to present the study findings.

\section{Results}

\section{Background characteristics of study participants}

A total of 22 individuals, 13 HCPs working in the management of NCDs and nine people with NCD, participated in this study. None of the purposively selected people to participate in this study have refused or dropped out. We tried to mix the participants from different backgrounds based on sex, age, the professional background of HCPs and NCD type for people with NCDs (see Tables 1 and 2).

Table 1

Background characteristics of HCPs working in NCD management, Addis Ababa, Ethiopia, April 2020

\begin{tabular}{|lllll|}
\hline Characteristics & \multicolumn{2}{l}{ Profession } & Total \\
\cline { 2 - 4 } & \multicolumn{2}{l}{ Doctors } & Nurses & Pharmacists \\
\hline Sex & & & & \\
\hline Male & 4 & - & 2 & 6 \\
\hline Female & 3 & 4 & - & 7 \\
\hline Age & & & & \\
\hline $20-30$ & - & - & - & - \\
\hline $31-40$ & 5 & 2 & 2 & 9 \\
\hline $41-50$ & 2 & 2 & - & 4 \\
\hline Service years & & & & 5 \\
\hline $10-15$ & 3 & - & 2 & 6 \\
\hline $16-20$ & 3 & 3 & - & 2 \\
\hline$>20$ & 1 & 1 & - & \\
\hline
\end{tabular}


Table 2

Background characteristics of people with NCD, Addis Ababa, Ethiopia, April 2020

\begin{tabular}{|c|c|c|c|c|}
\hline \multirow[t]{2}{*}{ Characteristics } & \multicolumn{3}{|c|}{ NCD types } & \multirow[t]{2}{*}{ Total } \\
\hline & Diabetes & Hypertension & Cancer & \\
\hline \multicolumn{5}{|l|}{ Sex } \\
\hline Male & 2 & 2 & 1 & 5 \\
\hline Female & 1 & 1 & 2 & 4 \\
\hline \multicolumn{5}{|l|}{ Age } \\
\hline $31-40$ & 1 & - & 1 & 2 \\
\hline $41-50$ & 1 & - & 1 & 2 \\
\hline $50-60$ & 1 & 3 & 1 & 5 \\
\hline \multicolumn{5}{|l|}{ Marital status } \\
\hline \multicolumn{5}{|l|}{ Single } \\
\hline Married & 1 & 2 & 2 & 5 \\
\hline Divorced & 1 & 1 & - & 2 \\
\hline Widowed & 1 & - & 1 & 2 \\
\hline \multicolumn{5}{|c|}{ Time since first diagnosis } \\
\hline$<5$ years & 4 & - & 2 & 6 \\
\hline $6-10$ years & 4 & 3 & - & 7 \\
\hline$>10$ years & 1 & 1 & - & 2 \\
\hline \multicolumn{5}{|c|}{ Challenges posed by COVID-19 for people with NCDs } \\
\hline \multicolumn{5}{|c|}{ Discontinuation of NCD follow-up } \\
\hline \multicolumn{5}{|c|}{$\begin{array}{l}\text { A } 52 \text {-year-old colorectal cancer patient said: '... I have been coming to this hospital for the past two years; it was always crowded, } \\
\text { now it looks like another hospital. I can say that there are no patients, it is quiet now'. All interviewed people with NCDs gave a } \\
\text { similar response. }\end{array}$} \\
\hline \multicolumn{5}{|c|}{$\begin{array}{l}\text { Almost all interviewed HCPs working in NCD management reported the same NCDs as the most common reasons for health } \\
\text { facility visits, admissions and death in the facility where they were working. They said that NCD patient flow to healthcare } \\
\text { facilities during COVID-19 had decreased drastically. They experienced a } 40-60 \% \text { decrease, especially during the first weeks after } \\
\text { notification of the first COVID-positive case in the country. }\end{array}$} \\
\hline $\begin{array}{l}\text { '...nowadays we } \\
\text { nephrologist). }\end{array}$ & ards at 1 & DVID we had be & morning & $1 P M^{\prime}(a$ \\
\hline
\end{tabular}

\section{Why people with NCD discontinue their follow-up during COVID-19}

Interview respondents with NCD gave varied reasons for the postponement of visits related to continuous NCD care, such as obtaining long-term treatment (NCD follow-up) during COVID-19. For example, a breast cancer patient who underwent follow-up for 3.5 years emphasised the lack of welcome at the healthcare facility: '...... This hospital was like my second home. I used to come here every time I need reassurance and emotional support. Now it is difficult to get into the hospital even with an appointment card, gate keepers are not allowing patients to pass in easily, plus we don't know if the repeated temperature measurements at the gate have side effects on us. These things discourage us from coming'.

Another cancer patient said: '...Public transport is not accessible nowadays; plus, the transport cost is double now, each passenger has to purchase the mandatory empty seat next to them...... so it is difficult to continue the follow-up as before'. However, those people with NCDs who own vehicles focused more on the fear of acquiring the virus within the facility.

Moreover, some said they were told by their healthcare providers not to come to healthcare facilities. 
All people with NCDs stated that they were under some form of stress in relation to COVID-19. They put forward a loss of social and economic support, fear of COVID-19 infection and the high risk of death as stressors and said that their relatives, neighbours and friends who used to visit and support them emotionally and financially before COVID-19 were not doing so after COVID-19.

'Families and friends had been visiting me before COVID-19, and they used to give me some money when they came, but now everybody is keeping their distance'(A typical response given by a colorectal cancer patient).

A 60-year-old diabetic patient said: 'My neighbours were the ones who were giving me my medications, but now everybody has left me; I can't take my medication by myself as I don't have my full vision... But I'm trying...'

'... We are repeatedly told that we are at higher risk of death from COVID-19. This information by itself, without clearly telling what we should do about it, is really stressful...'(Another diabetic patient).

\section{Decreased physical activity by people with NCD during COVID-19}

People with NCD who participated in this study reported their physical activity had decreased during COVID-19. For example, a diabetic patient from private clinic said: 'as we are told that we are at higher risk of dying from COVID-19, for fear of acquiring the virus I stayed fully at home. I even gained $7 \mathrm{~kg}$ within one month. I tried to do some exercise at home but I couldn't control the weight gain.'

A breast cancer patient said that she had even quit her job and stayed at home in order to minimise her risk from the virus. '...after I heard that we are at higher risk of death from COVID-19, I immediately quit my job. When I stayed at home, I tried to go out walking in the late afternoon, but now everybody is doing that and the walkways are crowded. Currently I'm not going out at all.'

\section{Challenges posed by COVID-19 to HCPs working on NCDs}

Health care professionals reported multiple challenges in NCD management during COVID-19. Some typical examples are as follows. 'Deciding on appointment dates is very stressful; we get into dilemmas in deciding on earlier appointment dates for better NCD management but higher risk of COVID-19 when they are coming to health care facilities, or a later appointment date with lower risk of COVID-19 but poor NCD management'(an endocrinologist).

In addition, most HCPs who participated in this study emphasised the shortage of personal protective equipment (PPE). 'We are worried that we don't have enough PPE; most attention is given to the COVID centres...but now, there is community transition and we can acquire the virus from any one of our patients...it is stressful...' (a nurse working at a primary healthcare facility)

From the HCPs' opinions, giving health education or not, especially for new diabetic patients, was a main challenge posed by COVID19 for the nurses working in a diabetic clinic. Deciding on the amount of medication to give, considering fair distribution for all in need and avoiding the person's risk of COVID from going out of the home to look for medication is another challenge for the pharmacists working in the dispensary. '...even if we have a stock of NCD medications for 3 months, I don't personally encourage that because there are a lot of problems; for example, our patients share their drugs and I doubt their storage too...'(a pharmacist).

The absence of clear direction and guidance is the biggest challenge for most of the HCPs who have participated in this study. '...as the pandemic is a new phenomenon for the world, we don't have manuals and guidelines; that makes the service delivery very challenging... but we are now developing those important documents with stakeholders...'(a senior oncologist).

\section{Coping strategies for healthcare professionals towards COVID-19 in the management of NCDs}

All HCPs said they have started virtual clinics by telephone. Many medical doctors call their NCD patients for follow-up; patients are asked about any complaints and whether they had any check-up records or investigation results. The aim was to replace the inperson check-up and to make the required clinical decisions.

However, almost all said that they had challenges in using the coping strategy designed for the phone clinic. They said that the use of a phone clinic was a new approach for them and that they were not given any training. Moreover, as they said, the phone clinic at which they were practicing had no guidelines or manuals. As a result, they missed many pertinent points and ended up with 
incomplete information. They also mentioned the absence of or an inaccessible contact address of patients as a challenge for the phone clinic.

Other measures adopted as a coping strategy in the management of NCDs during COVID-19 included the prescription of medication for longer times in order to avoid NCD patients having to leave home unnecessarily to replace their medication. However, people with NCD indicated that although they were given prescriptions for a longer time, it was difficult to get all the prescribed medication at once.

A chronic disease head nurse working in a primary health care facility said that they were following a community engagement strategy to address people with NCD at home '...As we have started house-to-house COVID-19 screening, we used this opportunity to ask for the presence of an NCD patient with health needs in the household...' Moreover, as said by an endocrinologist: 'messages were posted on social media for people with NCD to call their health care facilities to continue their follow-up. Contact address of HCPS were also communicated in the post...'

\section{Coping strategies for people with NCDs towards COVID-19 in the management of NCDs}

Few people with NCDs had identified their individual level coping strategies; for example, a hypertensive patient said: "I started to go to my office by walking, not using public transport...' Nevertheless, as per our study participants expression, the majority of them were expecting spiritual protection and safe guard.

\section{Discussion}

The findings of this study showed that the flow of NCD patients to healthcare facilities decreased during COVID-19. The WHO rapid assessment survey findings on service delivery for NCDs during the COVID-19 pandemic among 194 Ministries of Health also showed that $77 \%$ of countries reported that NCD services were disrupted [21]. However, it was suggested that those patients with NCDs are at higher risk of severe COVID-19 infection and need continuous monitoring and follow-up [20]. The WHO survey findings also showed that the more severe the transmission of the COVID-19 pandemic, the more were NCD services disrupted [21]. Therefore, as Ethiopia is in the earlier phase of the pandemic, we expect more NCD service disruptions in the future unless NCD-inclusive COVID-19 tackling strategies are executed. This could possibly lead to more NCD-related long-term health problems and even deaths.

Fear of acquiring COVID-19 infection was reported by most people with NCDs who participated in this study as a reason for discontinuation of NCD follow-up. Similarly, a study from Kenya showed that during the COVID-19 pandemic access to medical care was difficult due to overwhelmed health systems, lockdown, curfews and fear of infection [22]. Such fears need to be addressed by innovative health service approaches, such as separating the diverse patient flows to avoid patients with NCDs to meet those with febrile illnesses.

These study participants moreover repeatedly mentioned transportation problems as a reason for discontinuing NCD follow-up. In line with that, evidence has shown the impact on NCDs of COVID-19 response measures are complex; reduction of transport links alongside restricted access to primary health care units, pharmacies and community services all disrupt continuity of care for people with NCDs [23].

People with NCDs in this study said that they decreased their physical activity during COVID-19 and even mentioned the associated weight gain. Studies from Europe also showed that restrictive measures during the COVID-19 pandemic offered a challenge to maintaining appropriate levels of physical activity and accessing healthy food. Reduced physical activity, including travel to work and other places, exercise and sports for recreational purposes led to an increase in obesity [24].

Studies have indicated that one of the epidemiological risk factors for acquiring COVID-19 is being in healthcare facilities and hospitals in countries where the virus has been reported [3]. Unfortunately, because of the chronic nature of the disease, patients with NCDs need to visit the health facilities more often to take their medication, to receive treatment or for check-up appointments. This may make them more vulnerable to COVID-19 than the general population. This study also showed that HCPs are under stress in deciding on longer or shorter appointment dates in relation to introducing a lower or higher risk of COVID-19.

The WHO recommends that people with NCDs secure a one-month supply of their medication, or longer if possible [25]. Ethiopia has adopted the WHO recommendation and the Federal Ministry of Health recommends the same. However, participants in this study put

Page $7 / 10$ 
forward the irrational use and substandard storage of NCD drugs and shortage of stock of some NCD drugs as COVID-19-related challenges.

Healthcare professionals in this study reported a shortage of PPE as their main challenge in the continuation of routine NCD healthcare services during COVID-19. It is true that PPE shortage is a global health issue during the COVID-19 pandemic and has been quoted in different studies and reports [26, 27]. Currently, the supply of PPE in Ethiopia has improved.

According to our study participants, one of the commonly used coping strategies in NCD management was the phone clinic. Similarly, among countries reporting service disruptions, $58 \%$ of countries globally are now using telemedicine (advice by telephone or online) to replace in-person consultations; in low-income countries the figure is $42 \%$ [28]. More guidelines and instruction would be helpful for service providers.

\section{Strengths And Weaknesses Of The Study}

Firstly, the study examined the phenomenon of NCD management during COVID-19 from the perspective both of people with NCD and health care professionals (data triangulation). Secondly, use was made of the best data collection technique (phone based) under the current COVID-19 pandemic situation. That meant that study participants would comfortably share their experiences. This qualitative study revealed the challenges for people with NCDs at an early stage of the pandemic and thus captured only a momentary situation. Similar interviews after some time would probably show more coping strategies.

\section{Conclusion}

We concluded that the postponement of NCD follow-up, in addition to stress and reduced physical activity, may worsen the increasing mortality from preventable NCDs in the country. We recommend every COVID-19 response measures to target NCD health service independently.

\section{Abbreviations}

CHS: College of Heath Sciences, HCP: Healthcare professionals, IRB: Institutional Review Board, MERS: Middle East Respiratory Syndrome, MPH: Master of Public Health, NCD: Non-communicable diseases, PPE: personal protective equipment, SARS: Severe Acute Respiratory Syndrome, WHO: World health organization

\section{Declarations}

\section{Ethics approval and consent to participate}

Verbal consent to participate was obtained. Verbal consent was chosen because all the interviews were made through telephone. The Addis Ababa University College of Heath Sciences Institutional Review Board (CHS-IRB) has approved the verbal consent and the ethical clearance under protocol number 027/20/SPH.

\section{Consent for publication}

Not applicable

\section{Availability of data and material}

The interview guides, information sheet and consent form used for this study are available.

\section{Competing interests}

The authors declare that they have no competing interests

\section{Funding}

Funding was provided by University- and Hospital-partnerships GIZ Germany. The role of the funder in the study was to provide the required budget for the study. 


\section{Acknowledgements}

We would like to thank Addis Ababa University for reviewing the proposal, giving feedback and ensuring ethical clearance. MartinLuther-University for securing funds to the project. Our deepest gratitude also goes to the study participants for their time.

\section{References}

1. Ethiopia moh. Ethiopia confirmed the first case of COVID-19. March 13, 2020.

2. ECDC. COVID-19 situation update worldwide, as of 18 July 2020. 18 July 2020.

3. Sohrabi C, Alsafi Z, O’Neill N, Khan M, Kerwan A, Al-Jabir A, et al. World Health Organization declares global emergency: A review of the 2019 novel coronavirus (COVID-19). International Journal of Surgery. 2020.

4. Guan W-j, Ni Z-y, Hu Y, Liang W-h, Ou C-q, He J-x, et al. Clinical characteristics of coronavirus disease 2019 in China. New England Journal of Medicine. 2020.

5. WHO. Information note COVID-19 and NCDs. Https://www.who.int/publications-detail/clinical-management-of-severe-acuterespiratory-infection-when-novel-coronavirus-(ncov)-infection-is-suspected2019.

6. ISTITVTO SDS. Characteristics of COVID-19 patients dying in Italy Report based on available data on March 20th, 2020 March 2020.

7. World Health Organization. Report of the WHO-China Joint Mission on Coronavirus Disease 2019 (COVID-19) 16-24 February 2020

8. III. IDSC. Informe COVID-19 n 28. 04 de mayo de 2020.

Https://www.isciii.es/quehacemos/Servicios/vigilanciasaludpublicarenave/enfermedadestransmisibles/Paginas/informescovid19.aspx May 5, 2020.

9. Safiya Richardson ea. Presenting Characteristics, Comorbidities, and Outcomes Among 5700 Patients Hospitalized With COVID19 in the New York City Area. JAMA. 2020 Apr 22;323(20).

10. WHO. noncommunicable diseases country profiles 2018. 2018.

11. Institute EPH. Ethiopia STEPS report on risk factors for non-communicable diseaes and prevalence of selected ncds. December 2016.

12. WHO. Globocan 2018. International Agency for Research on Cancer 2018.

13. WHO. noncommunicable diseases progress monitor 2017. 2017.

14. Witter S, Zou G, Diaconu K, Senesi RG, Idriss A, Walley J, et al. Opportunities and challenges for delivering non-communicable disease management and services in fragile and post-conflict settings: perceptions of policy-makers and health providers in Sierra Leone. Conflict and Health. 2020;14(1):3.

15. Shiferaw F, Letebo M, Misganaw A, Feleke Y, Gelibo T, Getachew T, et al. Non-communicable Diseases in Ethiopia: Disease burden, gaps in health care delivery and strategic directions. Ethiopian Journal of Health Development. 2018;32(3).

16. Médecins Sans Frontières. COVID-19 pandemic brings new challenges to well-developed healthcare systems. 31 March 2020.

17. Ethiopia FDRO, Agency CS. Population Projection of Ethiopia for All Regions At Wereda Level from 2014 - 2017. August 2013.

18. Health Mo. Ethiopian Health Data Analytics Platform. Https://moh-public.ehdap.com/dashboard/non_communicable_diseases; 2010.

19. Network IGH, editor: Leaving No One Behind - Keeping up Essential Services for NCD Patients in Ethiopia during the Time of COVID-19 Response. Conversations on COVID-19 14th Webinar; June 19, 2020.

20. Fang L, Karakiulakis G, Roth M. Are patients with hypertension and diabetes mellitus at increased risk for COVID-19 infection? The Lancet Respiratory Medicine. 2020.

21. Department WN. final results Rapid assessment of service delivery for non communicable diseases (ncds) during COVID 19 pandemic. 18 June 2020 at 17:00.

22. Bosire EN, editor Kenya can't afford to neglect people with underlying conditions during COVID-19May 6, 2020 5.01pm SAST.

23. Kluge HHP, Wickramasinghe K, Rippin HL, Mendes R, Peters DH, Kontsevaya A, et al. Prevention and control of noncommunicable diseases in the COVID-19 response. Http://wwwthelancetcom/. May 8, 2020395. 
24. Europe wrof. Prevention and control of ncds at core of COVID-19 response. 17-06-2020.

25. World Health Organization. COVID-19 and ncds. 23 March 2020.

26. Malani P. Personal Protective Equipment Shortages During COVID-19-Supply Chain-Related Causes and Mitigation Strategies. JAMA. May 12, 2020.

27. Ranney ML, Griffeth V, Jha AK. Critical Supply Shortages - The Need for Ventilators and Personal Protective Equipment during the Covid-19 Pandemic. NEJM. March 25, 2020.

28. WHO. COVID-19 significantly impacts health services for noncommunicable diseases. 1 June 2020

\section{Supplementary Files}

This is a list of supplementary files associated with this preprint. Click to download.

- BezawitetalConsolidatedcriteria.docx

- Additionalfile1BezawitetalQuestionnaire.docx 